\title{
EQUINODERMOS DE AGUAS PROFUNDAS DE LA BAHLA TODOS SANTOS, BAJA CALIFORNIA
}

\author{
Por \\ Isaí Pacheco Ruiz y Luis F. Aguilar Rosas \\ Instituto de Investigaciones Oceanológicas \\ Universidad Autónoma de Baja California \\ Apartado Postal 453 \\ Ensenada, Baja California, México.
}

\section{RESUMEN}

En otoño de 1980 se realizaron muestreos bentónicos de fauna con draga Van Veen $y$ dos tipos de red de arrastre en 16 estaciones en la Babia Todos Santos (Fig. 1). Se detcrminó la ocurrencia de 7 organismos del Phylum Echinodermata de los cuales 3 fueron asteroideos, 2 ofiuroideos y 2 equinoideos. Además, se reporta un nuevo rango de distribución geográfico para Amphiodia occidentalis.

\section{ABSTRACT}

Bentbic faunistic samples were taken by a Van Veen dredge and two types of nets in 16 stations in Todos Santos Bay. The occurrence of 7 organisms of the Pnylum Echinodermata were determined; 3 asteroids, 2 opbiuroids and 2 ecbinoids. A new geograpbic distributional range for Amphiodia occidentalis is reported.

\section{INTRODUCCION}

Los estudios sistemáticos de las aguas oceánicas adyacentes a las costas, es uno de los aspectos más importantes en las tareas de investigación que actualmente se vicnen desarrollando. Las investigaciones oceanológicas pueden proporcionarnos el conocimiento adecuado de los recursos naturales existentes para asi contribuir el desarrollo de las técnicas de conservación y explotación de especies.

Las observaciones físico-químicas de la bahía no son escasas; se tienen datos sobre temperatura (Cabrera, 1974, Contreras, 1973; Morales, 1977); corrientes (Alvarez, 1971), vientos (Alvarez, 1977), y eventos de surgencias (Cota, 1971 y Chávez, 1975). Además existen datos de variaciones topográficas y cambios en la morfología de las playas (Secretaría de Marina, 1974 y Lizárraga, 1976).

En lo que respecta a observaciones biológicas, éstas sí son escasas, y la ma-

$$
\text { CIENCIAS MARINAS (Mex.) V. } 8 \text { (2), } 1982
$$


yoría han estado enfocadas a las zonas de entremareas (Secretaría de Marina, 1974, García, 1976; Devinny, 1978; González, 1979; Aguilar, 1981) y dirigidas a la flora algal de estos sitios. La única excepción es un trabajo sobre foraminíferos, realizado en zonas profundas de la Bahía de Todos Santos (Walton, 1955). Por lo anterior, se considera que este trabajo es una contribución al conocimiento de la fauna del Phylum Echinodermata de las zonas profundas de la Bahía Todos Santos.

\section{LOCALIZACION Y DESCRIPCION DEL AREA DE ESTUDIO}

La Bahía Todos Santos, Baja California, está localizada entre los $31^{\circ} 40^{\prime}$ y $31^{\circ} 55^{\prime} \mathrm{N}$ y $\operatorname{los} 115^{\circ} 36^{\prime}$ y $116^{\circ} 50^{\circ} \mathrm{W}$ (Fig. 1), y tiene un área aproximadamente de $167.6 \mathrm{~km}^{2}$. Está limitada al sur por Punta Banda, que es una zona montañosa con acantilados cortados verticalmente, al oeste por las Islas Todos.Santos y al norte por zonas rocosas con ocasionales playas de bolsillo. Posee un estero separado por una barra de arena que se extiende desde la parte sur de Punta Banda hacia el suroeste.

La bahía se considera poco profunda, ya que un 80 o/o del bentos se encuentra a menos de $50 \mathrm{~m}$ de profundidad y el 20 o/o restante forma parte de un cañón submarino localizado entre las islas y Punta Banda (Fig. 2).

Generalmente los vientos dominantes vienen del noroeste, con velocidad promedio de $4 \mathrm{~m} / \mathrm{seg}$, siendo en otoño e invierno cuando se presenta la mayor variación de la dirección del viento (Alvarez, 1971).

\section{METODOLOGIA}

Se determinaron 16 estaciones de colecta en la Bahía Todos Santos, para lo cual se tomó en cuenta que abarcaran la mayor variedad de condiciones ambientales como hidrología, sedimentos, corrientes, profundidad, grado de contaminación, etc., esto con el fin de obtener una mayor diversidad de hábitats de aguas profundas de la bahía. Cabe mencionar que para situar la red de estaciones con respecto a los parámetros antes citado, se consultaron los trabajos realizados por la Secretaría de Marina (1974), Cabrera (1974), Chávez (1975) y el Instituto de Investigaciones Oceanológicas de la Universidad Autónoma de Baja California (1979).

Las muestras fueron colectadas en 1980 , durante las êpocas del otoño, en las que se efectuaron 4 cruceros con una duración de 12 horas cada uno. Se tomaron 16 muestras con una draga Van Veen (Fig. 3) y entre cada una de las estaciones se llevaron a cabo 2 tipos de arrastre de aproximadamente 5 minutos cada uno, uno con la red tipo Nino y etro con un tipo de red de astre modificada (Figs. 4 y 5 ), haciendo un total de 24 arrastres bentónicos. 


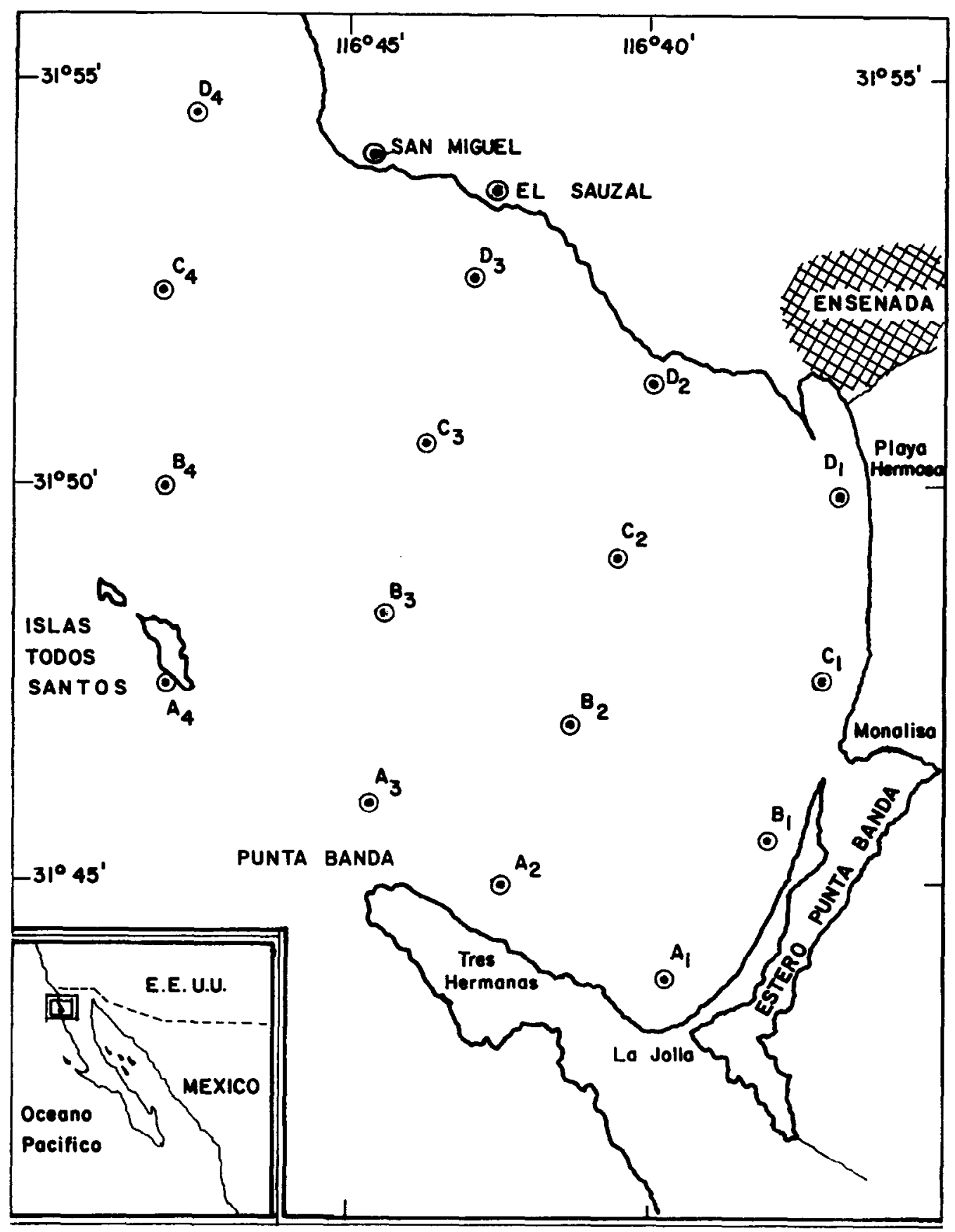

Fig. 1 Localización de las estaciones de colecta en La Bahía Todos Santos, Baja California. 


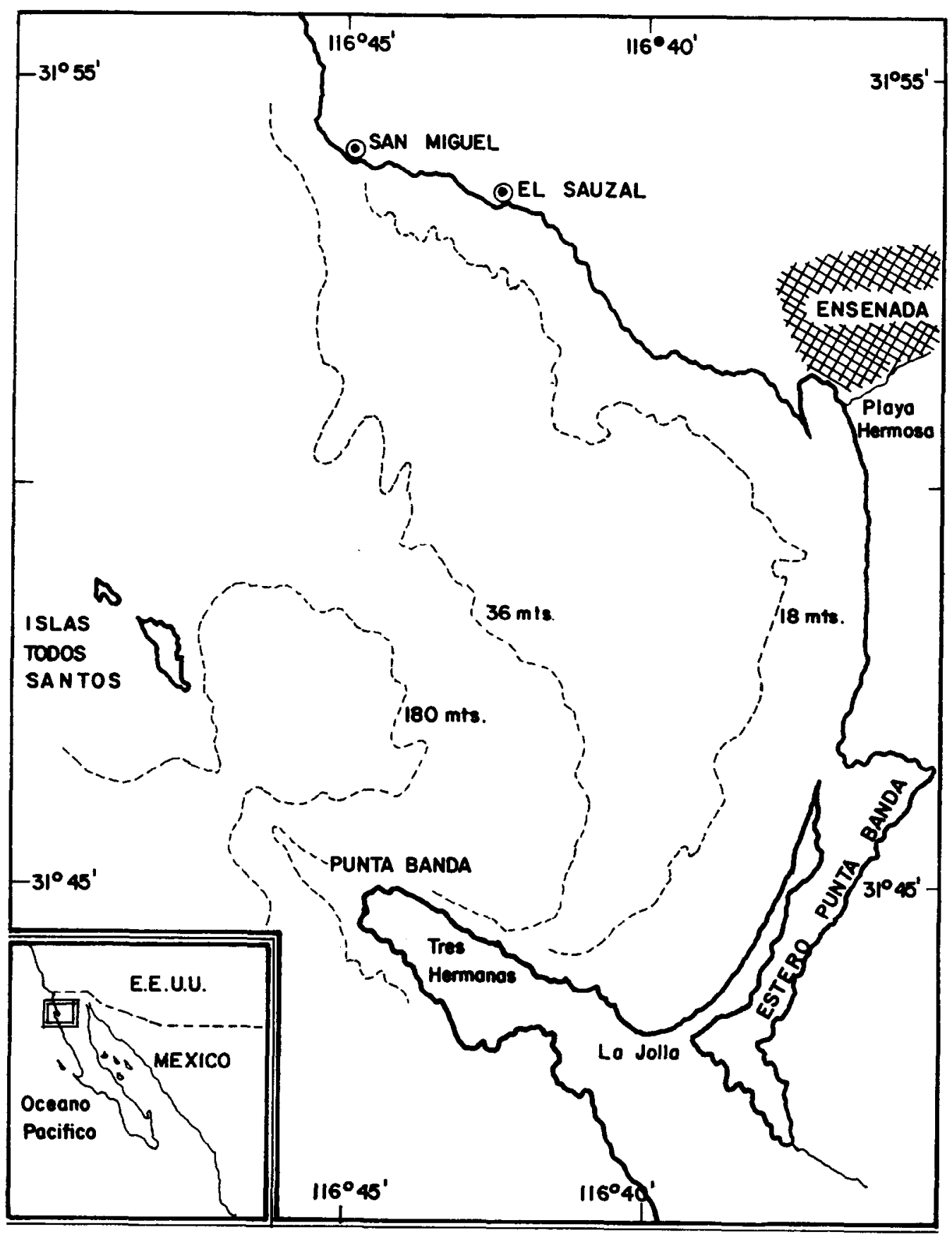

Fig. 2 Profundidades de La Bahía Todos Santos, B. C. 
Pacheco Ruiz -- Aguilar Rosas

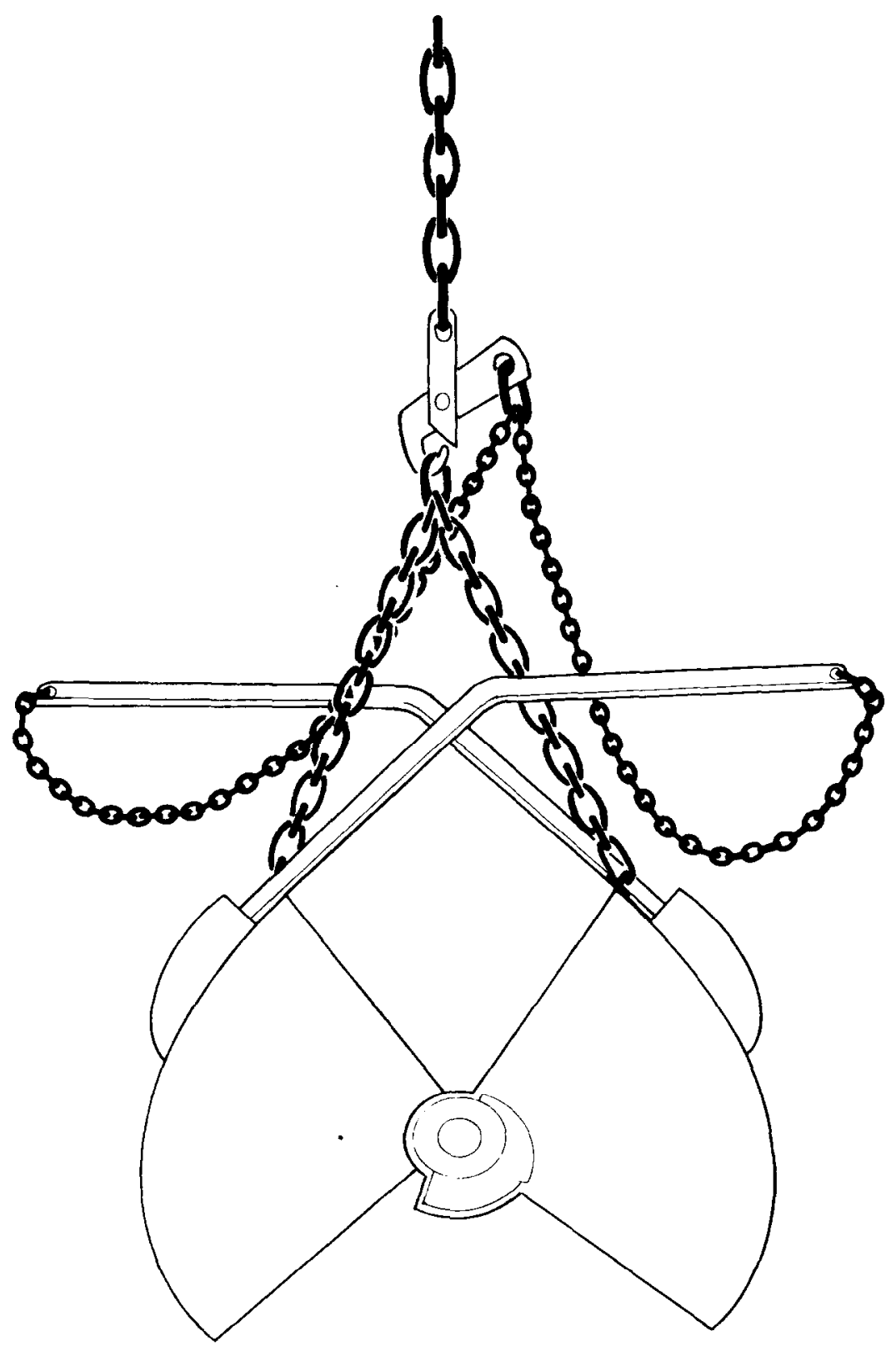

Fig. 3 Draga Van Veen. 
ECHINODERMOS DE LA BAHIA DE. TODOS LOS SANTOS

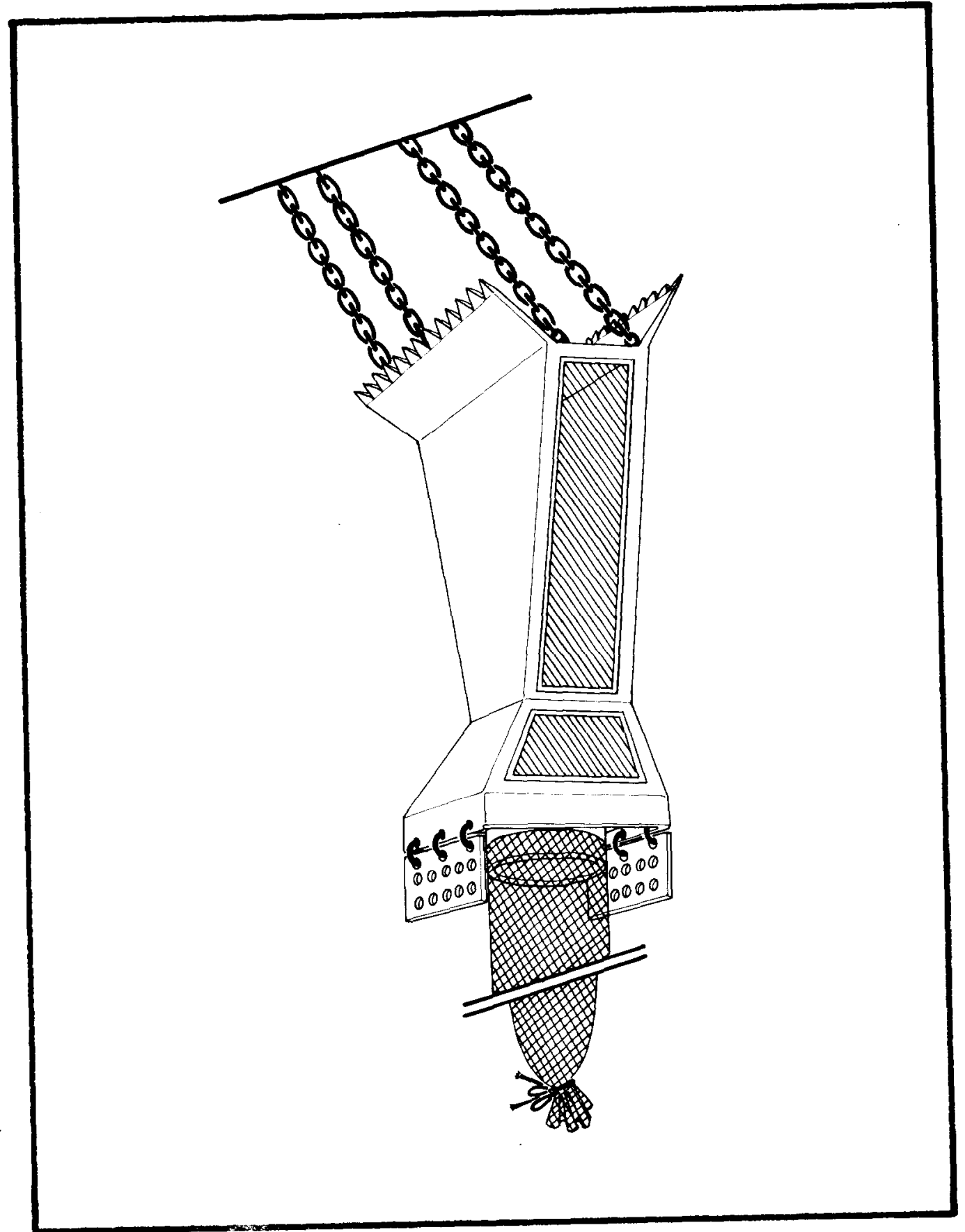

Fig. $4 \quad$ Red de arrastre tipo nino. 
Pacheco Ruiz - Aguilar Rosas

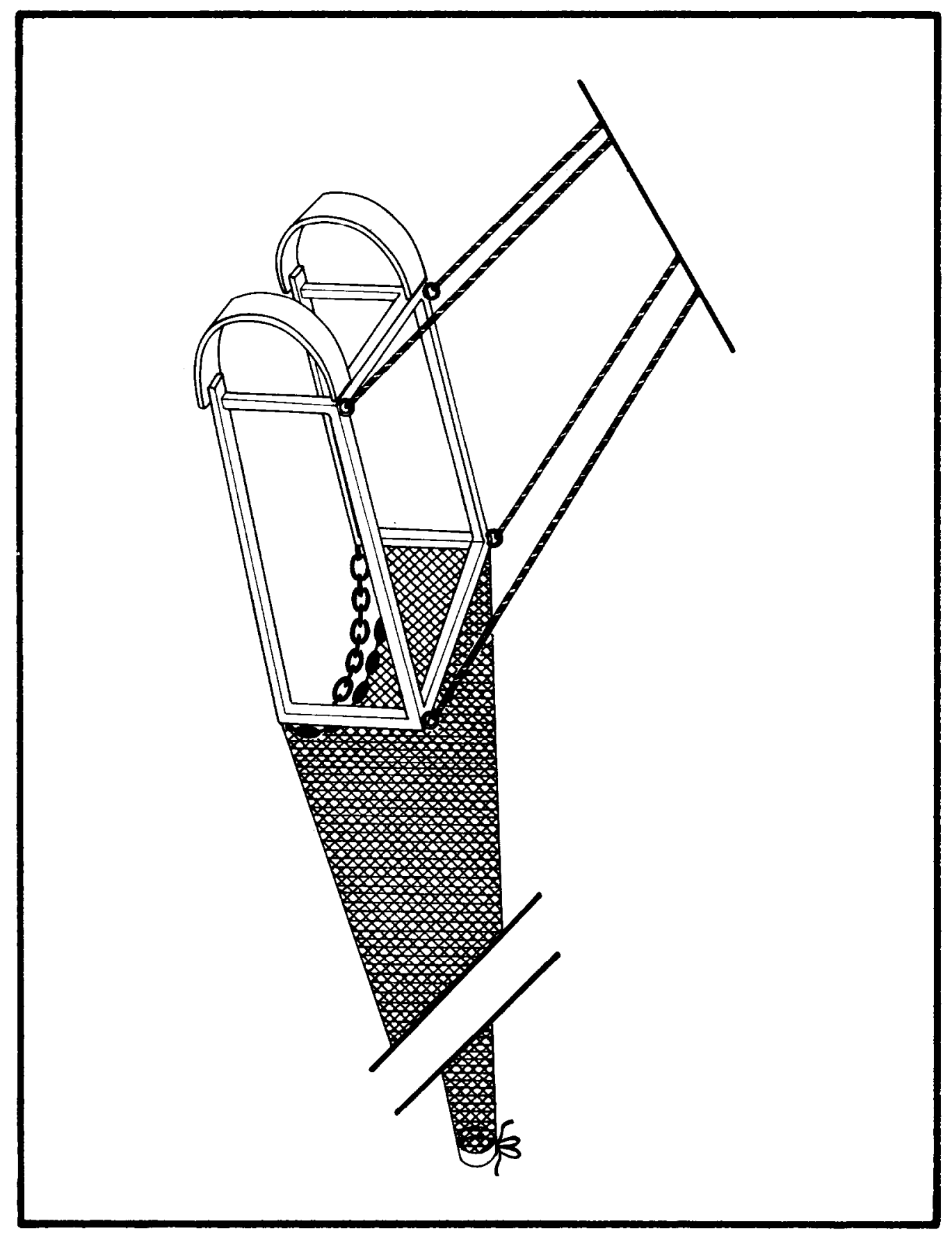

Fig. 5 Red de arrastre. 
Las profundidades de cada estación fueron determinadas por medio de la ecosonda de la embarcación y en algunas estaciones se midió la longitud del cablc de la draga y con un clinómetro se calculó la profundidad corroborándose así los datos anteriores.

Una vez colectados los organismos, se etiquetaron y preservaron con alcohol al 85 o/o para su posterior análisis en el laboratorio, donde fucron identificados de acuerdo a Morris, Abbot y Haderlie (1980) y Allen (1977).

\section{RESULTADOS}

En el presente trabajo se reportan 7 géneros del Phylum Echinodermata de la Bahía Todos Santos:

PHYLUM Echinodermata

CLASE Asteroidea

CLASF. Ophiuroidea

CLASE Echinoidea

$$
\begin{aligned}
& \text { Astropecten armatus } \\
& \text { Astropecten verrilli } \\
& \quad \text { californicus } \\
& \text { Patiria miniata } \\
& \text { Opbioderma panamense } \\
& \text { Ampbiodia occidentalis } \\
& \text { Lytecbinus anamesus } \\
& \text { Lovenia cordiformis }
\end{aligned}
$$

(Gray, 1840)

(Fisher, 1911)

(Brandt, 18.35)

(Lütken, 1859)

(Lyman, 1860)

(H.L. Clark, 1912)

(A. Agassiz, 1872)

En la Tabla I aparecen los ejemplares por estación y además se aprecia el tipo de técnica utilizada en los muestreos. En ésta se observa que en los transectos $\mathrm{A}$ y $\mathrm{D}$ se presenta mayor diversidad de especies y en $\operatorname{los} \operatorname{transectos} \mathrm{C}$ y $\mathrm{B}$, menor diversidad. Además de que en ella se menciona a una espécie (Ampbiodia occidentalis) con un nuevo rango de distribución geográfica.

Las profundidades y tipo de sustrato obtenidos para cada cstación se muestran en la Tabla II, donde se observa que la mayoría del sustrato en la bahía fuc arena, a excepción de las estaciones A4 y D3 donde sc encontró un sustrato rocoso.

\section{DISCUSIONES Y CONCLUSIONES}

Dada la carencia de estudios realizados sobrc aspectos de distribución de organismos de zonas profundas en la Bahía Todos Santos, consideramos el presente como un estudio básico, ya que se encontraron sólo 6 especies características de esas zonas. 
Tabla 1.- Especies colectadas en cada estación para cada tipo de muestreo.

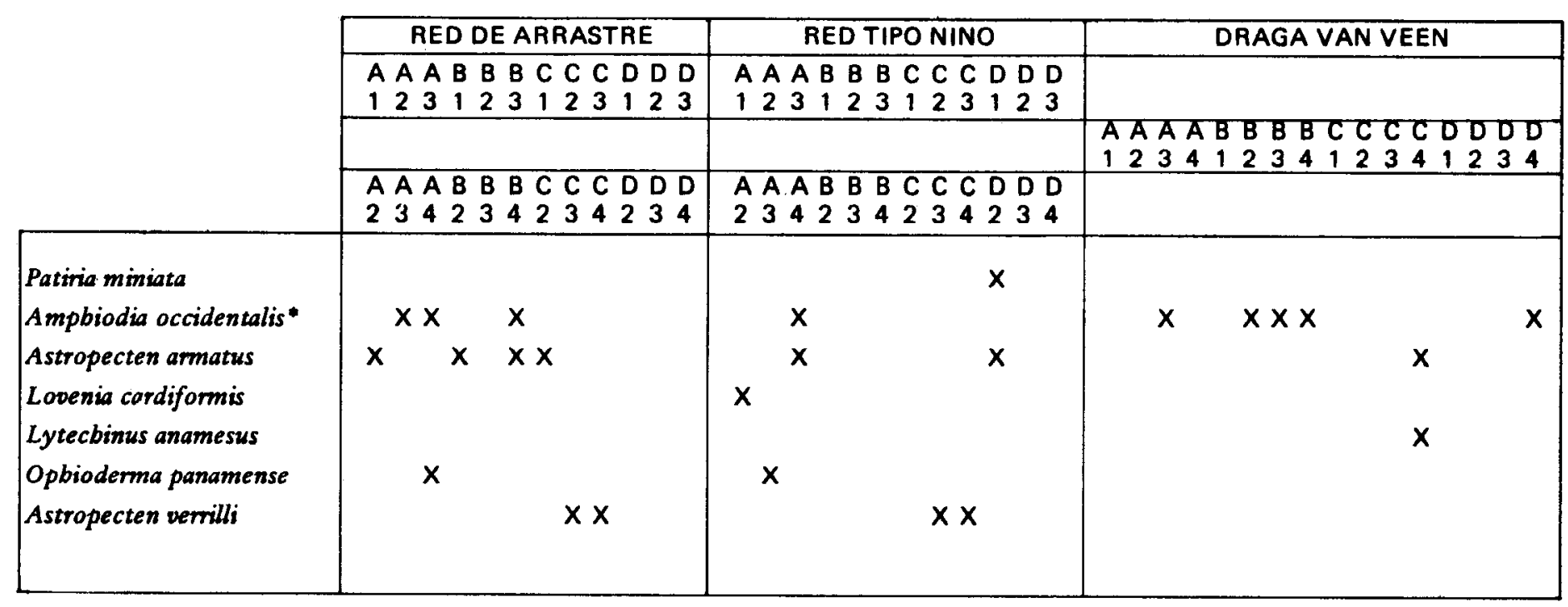

"Nuevo rango de distribución geográfica. 
Tabla II.- Tipos de sustrato y profundidades de cada estación de colecta

\begin{tabular}{|c|c|c|}
\hline ESTACION & PROFUNDIDAD & TIPO DE SUSTRATO \\
\hline A1 & $13 \mathrm{~m}$ & Arena fina de color verde pálido \\
\hline $\mathrm{A} 2$ & $51 \mathrm{~m}$ & Arena limosa de color verde oscuro \\
\hline A3 & $76 \mathrm{~m}$ & Arena fina de color verde gris \\
\hline A4 & $32 \mathrm{~m}$ & Arena gruesa con cantos rodados \\
\hline B1 & $8 \mathrm{~m}$ & Arena fina de color gris oscuro \\
\hline B2 & $36 \mathrm{~m}$ & Limo de color verde oscuro \\
\hline B3 & $60 \mathrm{~m}$ & Limo de color verde claro \\
\hline$B 4$ & $45 \mathrm{~m}$ & $\begin{array}{l}\text { Arena fina con fragmentos de concha; } \\
\text { color verde claro. }\end{array}$ \\
\hline $\mathrm{C} 1$ & $85 \mathrm{~m}$ & Arena fina de color gris oscuro \\
\hline $\mathrm{C} 2$ & $27 \mathrm{~m}$ & Limo de color verde claro \\
\hline C3 & $32 \mathrm{~m}$ & Arena fina de color verde pálido \\
\hline $\mathrm{C} 4$ & $49 \mathrm{~m}$ & Arena fina de color verde pálido \\
\hline D1 & $12 \mathrm{~m}$ & Limo de color verde oscuro \\
\hline $\mathrm{D} 2$ & $9 \mathrm{~m}$ & $\begin{array}{l}\text { Arena de tamaño mediano con frag- } \\
\text { mentos de concha; color amarillo } \\
\text { claro. }\end{array}$ \\
\hline D3 & $28 \mathrm{~m}$ & Grava \\
\hline D4 & $54 \mathrm{~m}$ & Arena gruesa de color oscuro \\
\hline
\end{tabular}


Fn base a los tipos de sustrato que se presentaron, consideramos que es un excelente hábitat para los organismos del Phylum Echonodermata que se han encontrato, ya que la mayoría de ellos se distribuyen en hábitats arenosos (Morris, Abbott y Haderlie, 1980).

En el caso de Opbioderma panamense y Patiria miniata colectados en sustratos arenosos, consideramos que aunque es más común encontrarlos en hábitats rocosos, el amplio rango de distribución en cuanto a la profundidad (Morris, Abbot y Haderlie, 1980), les permite tener mayor habilidad de colonizar otros tipos de sustrato.

Todos los ejemplares colectados a excepción de $O$. panamense se localizaron dentro de los rangos de profundidad establecidos. En el caso de $O$. panamense, se encontró que rebasó los rangos de profunidad reportados, según Morris, Abbot y Haderlie (1980), ya que el rango era desde las zonas de entremareas hasta $40 \mathrm{~m}$ de profunidad. En la bahía se colectó en varias ocasiones hasta los $70 \mathrm{~m}$ de profundidad. Aunque cabe la posibilidad de que $O$. panamense haya sido transportado accidentalmente por corrientes a lugares más profundos, esta disminuye, dado lo común de su ocurrencia en esa profunidad.

Es importante mencionar que en el caso de Ampbiodia occidentalis se ha ampliado su rango de distribución ya que su localización anterior mas al sur en la costa Pacífica era San Diego, California, E.E.U.U., y con este trabajo se extiende su rango de distribución sur hasta la Bahía Todos Santos, Baja California, México.

\section{LITERATURA CITADA}

AGUILAR ROSAS, L.E., 1981. Algas rojas (Rhodophyta) de la Bahía Todos Santos, Baja California, México. Ciencias Marinas, 7 (1):85-101.

ALLEN, R.K., 1977. Common intertidal Invertebrates of Southern California. Peek Publications. 316 pp.

ALVAREZ SANCHEZ, L.G., 1971. Mediciones de corrientes superficiales en la Bahía Todos Santos, Baja California. Tesis de Licenciatura, Escuela Superior de Ciencias Marinas, Universidad Autónoma de Baja California. Ensenada, B.C. 54 pp.

1977. Vientos de la Bahía Todos Santos, Baja California. Ciencias Marinas, $4(1): 81-89$.

CABRERA MURO, H.R., 1974. Distribución de temperatura en la Bahía Todos Santos. Ciencias Marinas. 1(1):65-77.

CHAVEZ GARCIA, M. del C., 1975. Algunas condiciones de surgencia durante la primavera de 1974 para el área adyacente a Punta Banda, Baja California. Ciencias Marinas 2(2):111-124.

CONTRERAS RIVAS, I., 1973. Influencia termohalina del estero en la Bahía Todos Santos, B. C. Tesis de Licenciatura, Escuela Superior de Ciencias Marinas, Universidad Autónoma de Baja California, Ensenada, B.C. 68 pp. 
COTA VILLAVICENCIO, A., 1971. Estudio oceanográfico químico de la surgencia localizada en la zona de Punta Banda, B.C. Tesis de Licenciatura, Escuela Superior de Ciencias Marinas, Universidad Autónoma de Baja California, Ensenada, B.C. 63 pp.

DEVINNY, J.S., 1978. Ordination of seaweeds communities. Enviromental gradients at Punta Banda, B.C., Mexico. Botánica Marina, XXI, Fasc. G., 357 $-363$.

GONZALEZ LOPEZ, J., 1979. Ficoflora litoral de la región de Ensenada, B.C. Tesis Profesional. Facultad de Ciencias, U.N.A.M., 174 pp.

INSTITUTO DE INVESTIGACIONES OCEANOLOGICAS, 1979. Estudios Básicos para el diagnóstico permanente de la contaminación de Baja California. Informe anual del Instituto de Investigaciones Oceanológicas, Universidad Autónoma de Baja California.

LIZARRAGA ARCINIEGA, J.R., 1976. Variaciones estacionales de la playa de la Bahía de Todos Santos, B.C. Ciencias Marinas, 3(1):30-50.

MORALES ZUNIGA, C., 1977. Variaciones estacionales de la temperatura en la Bahía de Todos Santos, B.C. Tesis de Licenciatura, Escuela Superior de Ciencias Marinas, Universidad Autónoma de Baja California, Ensenada, B.C. $56 \mathrm{pp}$.

MORRIS, R.H. D.P., Abbott and E. Haderlie, 1980. Intertidal Invertebrates of California. Stanford University Press, Stanford California. $690 \mathrm{pp}$.

SECRETARIA DE MARINA, 1974. Estudio Geográfico de la región de Ensenada, Baja California. Dirección General de Oceanografía y Señalamiento Marítimo.

WALTON, W.R. , 1955. Ecology of living Benthonic Foraminifera. Todos Santos Bay. J. of Paleontology 29(6):952-1018. 